\title{
ARTICLE OPEN Spliceosome protein Eftud2 promotes colitis-associated tumorigenesis by modulating inflammatory response of macrophage
}

\author{
Zhonglin Lv ${ }^{1}$, Zhihong Wang ${ }^{1,2}$, Longlong Luo ${ }^{1}, \mathrm{Yu} \mathrm{Chen}^{3}$, Gencheng Han ${ }^{4}$, Renxi Wang ${ }^{4}$, He Xiao ${ }^{1}$, Xinying $\mathrm{Li}^{4}$, Chunmei Hou ${ }^{4}$, \\ Jiannan Feng ${ }^{1}$, Beifen Shen ${ }^{4}$, Yi Wang ${ }^{5}$, Hui Peng ${ }^{2,6}$, Renfeng Guo ${ }^{7}$, Yan Li ${ }^{4}$ and Guojiang Chen ${ }^{1}$
}

\begin{abstract}
Alternative splicing (AS) of mRNA is known to be involved in regulation of immune cell differentiation and activation. Elongation factor Tu GTP binding domain containing 2 (Eftud2) is an AS factor to potentially modulate innate immune response in macrophages. In this study, we investigate its involvement in the pathogenesis of colitis-associated cancer (CAC). Using an established mouse model of CAC, we show that Eftud2 is constantly overexpressed in the colonic tissues as well as infiltrating macrophages. Myeloid-specific knockout of Eftud2 remarkably suppresses chronic intestinal inflammation and tumorigenesis, which is associated with decreased production of inflammatory cytokines and tumorigenic factors. Repression of colonic inflammation and colorectal tumor development in Eftud2-deficient mice is due to the impaired activation of NF-KB signaling in LPS-challenged macrophages. Furthermore, the alteration of Eftud2-mediated AS involving the components of TLR4-NF-KB cascades underlies the impairment of NF-KB activation. Overall, these findings provide new insights into the tight link between inflammation and cancer and modulation of AS in innate immune signals may be a potentially therapeutic avenue for CAC treatment.
\end{abstract}

Mucosal Immunology (2019) 12:1164-1173; https://doi.org/10.1038/s41385-019-0184-y

\section{INTRODUCTION}

Colorectal cancer is the third most common form of cancer and the second leading cause of cancer-related death in developed countries. ${ }^{1}$ Notably, patients with inflammatory bowel diseases (IBD) such as Crohn's disease and ulcerative colitis are at increased risk for the development of colorectal cancer. ${ }^{2}$ Although the precise molecular mechanism of IBD-related colorectal tumor formation is incompletely understood, it is widely viewed that a series of cytokines and chemokines produced during chronic inflammation contribute to malignant transformation. ${ }^{3}$

The chronic inflammatory microenvironment is characterized by infiltration with mononuclear cells, and macrophages are the dominant cellular players in chronic inflammation. ${ }^{4}$ Lamina propria macrophages represent a major cellular source of several proinflammatory cytokines (e.g., IL-6, TNF- $a$, and IL-1 $\beta$ ) in IBD and play a key role in the pathogenesis of colitis and the associated tumorigenesis. ${ }^{5-7}$ In IBD-related tumorigenesis, one of the unique aspects of colorectal cancer development is the involvement of lumenal flora and TLR4 signaling. ${ }^{8}$ The engagement of TLR4 with its ligand LPS leads to macrophage activation involving multiple innate immune receptors and kinases, among which the downstream transcription factor NF-KB is a central mediator of proinflammatory cytokine production. ${ }^{9}$
Macrophage activation involves widespread transcriptomic changes, reflecting regulation via transcription, and mRNA decay, ${ }^{10}$ yet alternative splicing (AS) is increasingly being recognized as important. ${ }^{11}$ The AS results in mRNAs that can encode protein isoforms with altered, antagonistic, or unrelated functions. Notably, LPS-activated mouse macrophages slow down splicing in certain pre-mRNAs thus favoring AS. ${ }^{12}$ Several splicing factors have been demonstrated to regulate TLR signaling and macrophage activation. ${ }^{13-15}$ Among these factors, the mRNA splicing regulator Eftud2, whose mutation causes a multiple malformation syndrome termed to mandibulofacial dysostosis with microcephaly, ${ }^{16}$ was recently shown to control the alternate splicing of the MyD88 adaptor to modulate the extent of the innate immune response in murine macrophages. ${ }^{17}$ However, the precise role of Eftud2 in chronic intestinal inflammation and the associated tumorigenesis is yet unknown.

\section{RESULTS}

Eftud2 expression is elevated significantly in mice suffering from colitis-associated cancer (CAC) and colitis

First, we collected the colon samples of mice suffering from CAC. Western blotting analysis showed that the expression of Eftud2

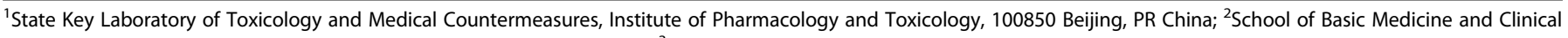

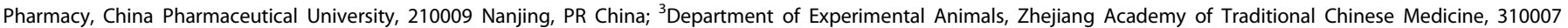

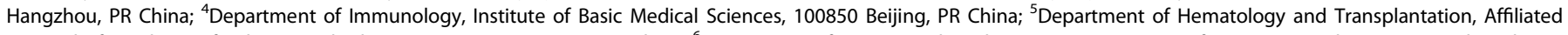

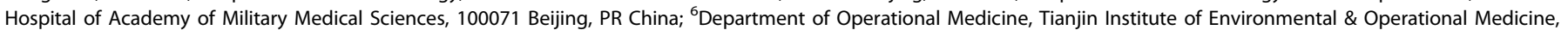
300050 Tianjin, PR China and ${ }^{7}$ Department of Pathology, University of Michigan, Ann Arbor, MI 48109, USA

Correspondence: Yu Chen (blow2000@163.com) or Guojiang Chen (jyk62033@163.com)
}

Received: 14 February 2019 Revised: 11 June 2019 Accepted: 12 June 2019

Published online: 5 July 2019 
protein increased significantly in the lesions of CAC-bearing mice compared with untreated littermates (Fig. 1b). Moreover, compared with nontumor colon tissues, tumors from the same recipients contained higher Eftud2 contents (Fig. 1c). Since it has been previously shown that Eftud2 has potentials to regulate innate immune responses, ${ }^{17}$ we next sought to determine the alteration of its expression in a model of colonic inflammation. We established an acute colitis model by drinking 3\% DSS. As expected, the levels of Eftud2 in the inflamed colon tissues, indeed, were elevated dramatically (Fig. 1d). These findings suggest that Eftud2 may play a role in intestinal inflammation and the associated tumorigenesis. We further determined the cellular sources of Eftud2 during CAC formation. Immune cell subsets and fibroblasts as well as intestinal epithelial cells were fractionated, respectively. Quantitative RT-PCR analysis showed that macrophages were the major source of Eftud2 and that Eftud2 expression substantially increased in tumor-associated macrophages (Fig. 1e). The localization of Eftud2 in infiltrating macrophages from the naïve (day 0 post $A / D$ ) and inflamed colons (day 35 post $A / D$ ) as well as tumor tissues (day 100 post A/D) was also identified by immunofluorescence staining (Fig. 1f). To further determine the link between Eftud2 expression and the inflammatory responses of macrophages, a murine macrophage line RAW264.7 was utilized to be stimulated with LPS. Eftud2 expression was increased in LPS-challenged macrophages in a dose and time-dependent manner (Fig. S1A, B). In parallel, an increase in IL- 6 production by macrophages following LPS stimulation was observed (Fig. S1A, B). Furthermore, we isolated macrophages residing in lamina propria of the colons and spleen of CAC-bearing mice, respectively. Compared with untreated controls, Eftud2 expression was increased significantly in macrophages from the proximal compartment (i.e., LP) to malignancies, while its expression in macrophages from the distant compartment (i.e., spleen) did not change (Fig. S1C). A similar trend in IL-6 expression in macrophages was observed (Fig. S1C). Given that IL-6 is recognized as a putatively tumor-promoting factor during CAC pathogenesis, ${ }^{5,18}$ it is conceivable that Eftud 2 may play a role in intestinal inflammation-associated tumorigenesis.

\section{Eftud2 deletion in macrophages suppresses colitis-associated} tumorigenesis

To directly determine the role of Eftud2 in macrophages during colon carcinogenesis, we generated myeloid-specific Eftud2-knockout mice by crossing LysM-Cre mice and Eftud2 $2^{\mathrm{f} / \mathrm{fl}}$ littermates (Fig. S2A; termed to MKO). Eftud2 deletion in macrophages isolated from bone marrow and peritoneal cavity was confirmed (Fig. S2B, C). Firstly, we sought to determine the possible role of Eftud2 in myelopoiesis. No significant alterations of the frequencies of monocytes (Ly-6C ${ }^{\text {hi }}$ CD $\left.11 b^{+}\right)$and granulocytes $\left(\right.$Ly- $6 G^{\text {hi }}$ CD $\left.11 b^{+}\right)$, as well as LK (myeloid progenitors, lin ${ }^{-} \mathrm{CD} 127^{-} \mathrm{ckit}^{-} \mathrm{sca}^{-}{ }^{-}$) and LSK cells (hematopoietic stem cells, lin $^{-} \mathrm{CD} 127^{-} \mathrm{c}^{-} \mathrm{kit}^{+} \mathrm{sca}-1^{+}$) were found in naïve MKO mice (Fig. S3A, B). Secondly, we isolated monocytes from bone marrow of WT and MKO mice and stimulated with M-CSF for 7 days. The pattern of the expression of monocyte's marker Ly- $6 C$ and macrophage's marker F4/80 during differentiation of monocytes into macrophages was comparable between WT and MKO immune cells (Fig. S3C), indicating a dispensable role of Eftud2 in macrophage development.

We next established AOM/DSS-induced CAC model in MKO and WT (Eftud2 ${ }^{\mathrm{f} / \mathrm{fl}}$ ) mice. MKO mice had significantly decreased tumor multiplicity and tumor burden in the colon (Fig. 2a, b). Further, MKO mice were significantly protected from development of highgrade dysplasia, developing a maximum of low-grade dysplasia (Fig. 2c). Representative HE-stained images demonstrated the decrease in tumor size and protection from high-grade dysplasia in MKO mice (Fig. 2d). Consistent with these macroscopic changes, increased apoptosis of colonic epithelial cells (CEC) was visible in MKO mice, evidenced by augmented cleavage of caspase 3
(Fig. 2e). This may be due to lower contents of Bcl-XL in neoplastic CEC of MKO mice than that of WT littermates (Fig. 2f), an antiapoptotic protein that is reported to mediate CEC survival. ${ }^{19}$ Moreover, the proliferation of CEC was limited in MKO mice, as reflected by reduced expression of Ki67 in CEC (Fig. 2e). Taken together, these data support a tumor-promoting role of Eftud2 in colon tumorigenesis.

Eftud2 loss in macrophages impairs the innate immune response in colon tumors

It is well accepted that the AOM/DSS model utilizes inflammation to drive tumorigenesis. Based on decreased tumor multiplicity and burden in MKO mice, we speculate that Eftud2 deletion in macrophages renders the impairment of immune responses to inflammatory stimuli or luminal insults. To address this issue, survival of mice subjected to AOM/DSS was monitored. MKO mice exhibited significant protection from intestinal injury-related mortality, as compared with WT mice (Fig. 3a). In conjunction with improved survival, MKO mice demonstrated significantly decreased histologic colitis with AOM/DSS versus WT mice (Fig. 3b, c). Furthermore, we dissected the colons of MKO and WT mice on day 35 and 100 post AOM/DSS instillation, respectively, and detected the expression of a series of proinflammatory cytokines and growth factors, which have been reported to contribute to CAC development. $^{20}$ The production of proinflammatory cytokines, including IL6 , TNF- $\alpha$, IL-1 $\beta, I L-22$, and IL-23, was all found to be reduced in the colons of MKO mice versus that in WT mice (Fig. 3d, e). Similarly, the colons of MKO mice contained lower levels of PGE2, Arginase-1 (Arg-1), inducible NO synthase (iNOS), GM-CSF, and M-CSF (Fig. 3e). Notably, some putatively CAC-promoting cytokines, including IL-1, IL-6, and TNF-a, play a pivotal role in STAT3 activation, a critical modulator of tumor-promoting chronic inflammation. ${ }^{21}$ We therefore assessed the activation status of STAT3 in our system. Augmented phosphorylation of STAT3 in CEC of CAC-bearing WT mice was observed, while STAT3 activation was abolished in CEC of MKO littermates (Fig. 3f). Intriguingly, the similar alterations of STAT3 activation were visible at the stage of inflammation (on day 35 post AOM/DSS) (Fig. 3f), in agreement with the established role of STAT3 in enhancing intestinal cell proliferation during colitisassociated tumorigenesis. ${ }^{5}$

To directly address the effect of Eftud2 deletion on the release of proinflammatory cytokines by macrophages, bone marrowderived macrophages (termed to BMDM) were stimulated with LPS for 12-24 h. IL-6, TNF- $a$, and IL-1 $\beta$ expression was significantly decreased in MKO macrophages (Figs. $3 \mathrm{~g}$ and S4). Taken together, these data indicate a key role of Eftud2 in mounting inflammatory immune reactions in the intestine during CAC development.

\section{Myeloid Eftud2 deficiency has no impact on trafficking of} macrophages

The chronic inflammation is characterized by chemokinedependent recruitment of innate immune cells, including granulocytes and macrophages, and thereafter release of large amounts of proinflammatory cytokines and mediators. Therefore, we address whether Eftud2 deletion has an effect on the chemotaxis of immune cell populations. The number of neutrophils and macrophages in tumors of MKO mice was comparable to those of WT mice (Figs. 4a and S5A). Furthermore, no difference in trafficking of other immune subsets such as $T$ and $B$ lymphocytes was observed between WT and MKO mice (Fig. S5A). Similar phenotypes were found at the stage of colitis (day 35 post $A / D$ ) (Figs. 4a and S5A). To further determine the effect of Eftud2 on macrophage trafficking, we established a model of peritonitis by injection of thioglycollate. We found that Eftud2 deletion did not affect the recruitment of macrophages into the site of inflammation (Fig. S6A). Since the trafficking of immune cells is regulated by the engagement of appropriate chemokines with their receptors, we next detected the levels of several chemokines in the colons. 


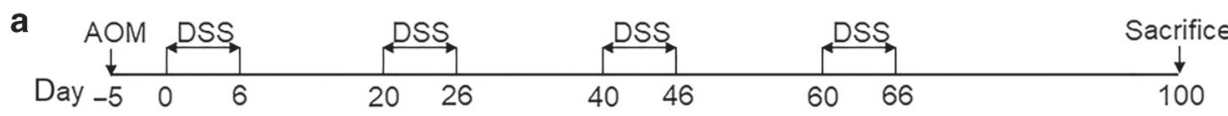

b

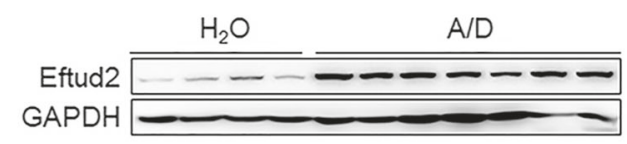

d

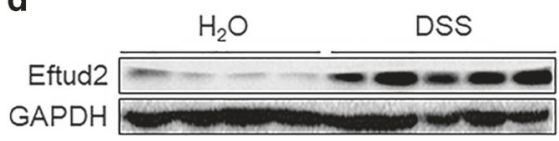

c

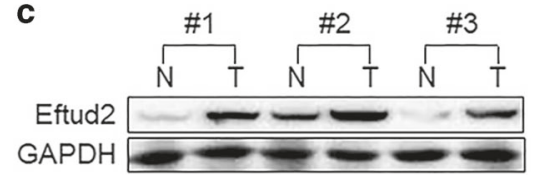

e

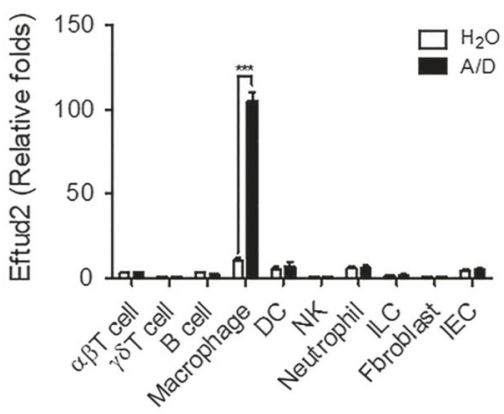

f DAPI CD68 Eftud2 Merge

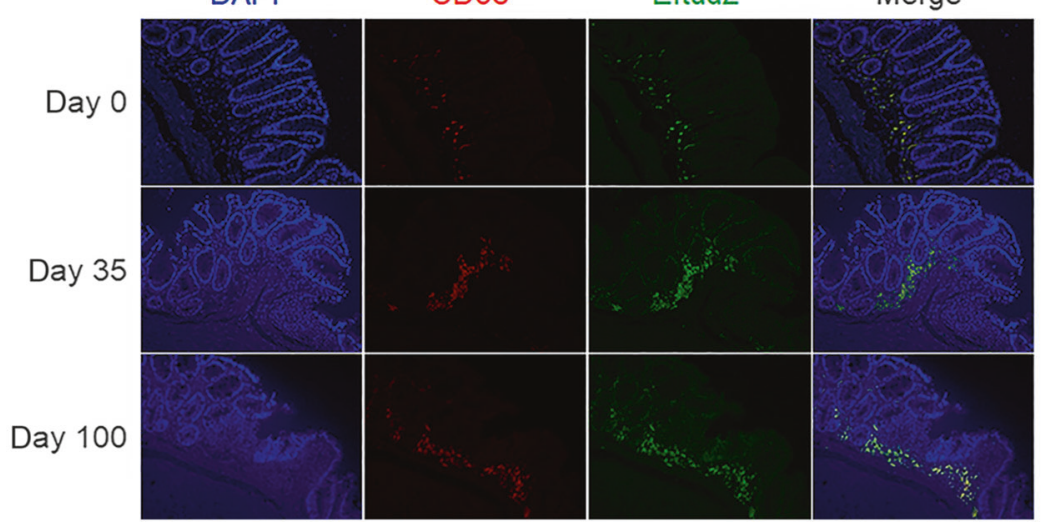

Fig. 1 Up-regulation of Eftud2 expression in CAC lesions as well as macrophage infiltrates. a Schematic overview of CAC protocols. In brief, mice were injected intraperitoneally with AOM $(10 \mathrm{mg} / \mathrm{kg})$. After 5 days, these rodents received drinking water containing $1.5 \%$ DSS for 1 week. Thereafter, mice were maintained on regular water for 2 weeks and received three more DSS treatment cycles. b On day 100 after CAC induction, Eftud2 expression in the colons was examined by Western blotting. Each lane represents one mouse. c Eftud2 expression in the tumor $(\mathrm{T})$ and non-tumor $(\mathrm{N})$ colon tissues of CAC-bearing mice was examined by Western blotting. The number indicates one mouse. $\mathbf{d}$ On day 15 after colitis induction, Eftud2 expression in the colons was examined by Western blotting. Each lane represents one mouse. e On day 100 following CAC induction, immune cell subsets in the LP of colons of MKO and WT mice were fractionated by FACS sorting and Eftud2 expression was examined by quantitative RT-PCR. Five pairs of mice were analyzed. $\mathbf{f}$ On day 0,35 , and 100 after CAC induction, the colon tissues of MKO and WT mice were dissected, respectively, and CD68 as well as Eftud2 staining was examined by immunofluorescence. Five pairs of mice were examined. An example is shown. Magnification: $\times 200$. ${ }^{* * *} P<0.001$

The expression of neutrophil-recruiting chemokines (CXCL1/2) and macrophage-recruiting chemokines (CCL2/7) did not change in MKO mice compared with WT controls (Fig. 4b, c). As well, a comparable level of other immune cell-recruiting chemokines (CXCL9/10/13) was found between MKO and WT mice (Fig. S5B). We further detected the CCR2 expression on macrophages in the model of peritonitis. Compared with circulating macrophages with a low level of CCR2 expression, those infiltrating into inflammatory compartment highly expressed this receptor, while Eftud2 deletion had no impact on its expression (Fig. S6B). This concept was further confirmed by detection of CCR2 expression on LPSchallenged BMDM ex vivo (Fig. S6C). Therefore, we can conclude that lack of Eftud2 does not influence macrophage and other immune cell trafficking to inflamed tissues.

It is known that one of arms of macrophages to exert antitumor functions is phagocytosis. We next assess whether tumor remission in Eftud2-MKO mice is due to the augmented capacity of engulfment to invading pathogens and cancer cells. As a result, Eftud2 deficiency did not affect phagocytosis of fluorescencelabeled E. coli in vitro (Fig. S7A). In addition, the survival of macrophages after serum depletion was decreased but not different in presence or absence of Eftud2 (Fig. S7B).

Eftud2 deletion in macrophages does not affect angiogenesis in colon tumors

Angiogenesis is a hallmark of carcinogenesis, and is an essential mean by which colon tumor growth is supported. ${ }^{22,23}$ Several cytokines, including vascular endothelial growth factor (VEGF) A and CXCL1, contribute to angiogenesis. ${ }^{24-26}$ Macrophages are important source of these proangiogenic factors. ${ }^{27,28}$ Based on decreased tumor multiplicity/burden and decreased macrophage activation in MKO mice, we determined whether angiogenesis was impaired, contributing to diminished tumorigenesis. As described above, CXCL1 expression was increased in CAC-bearing mice, but 

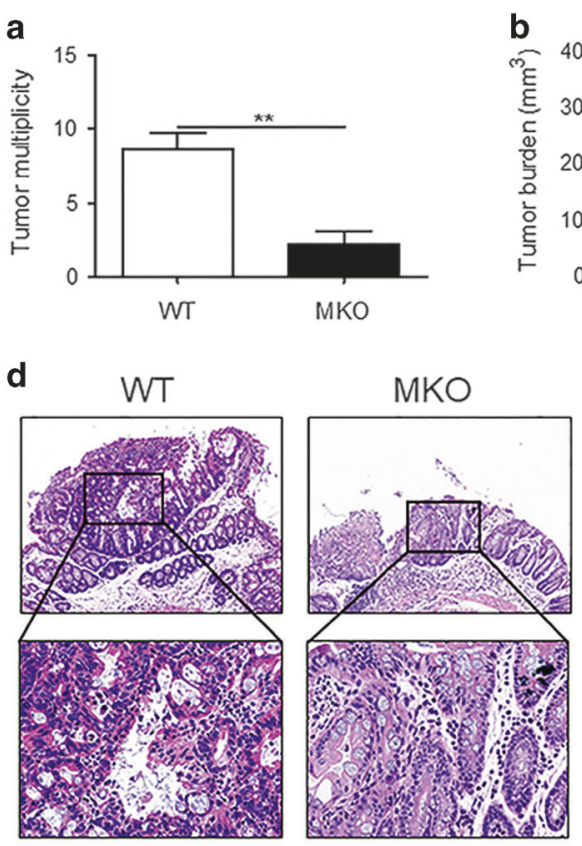
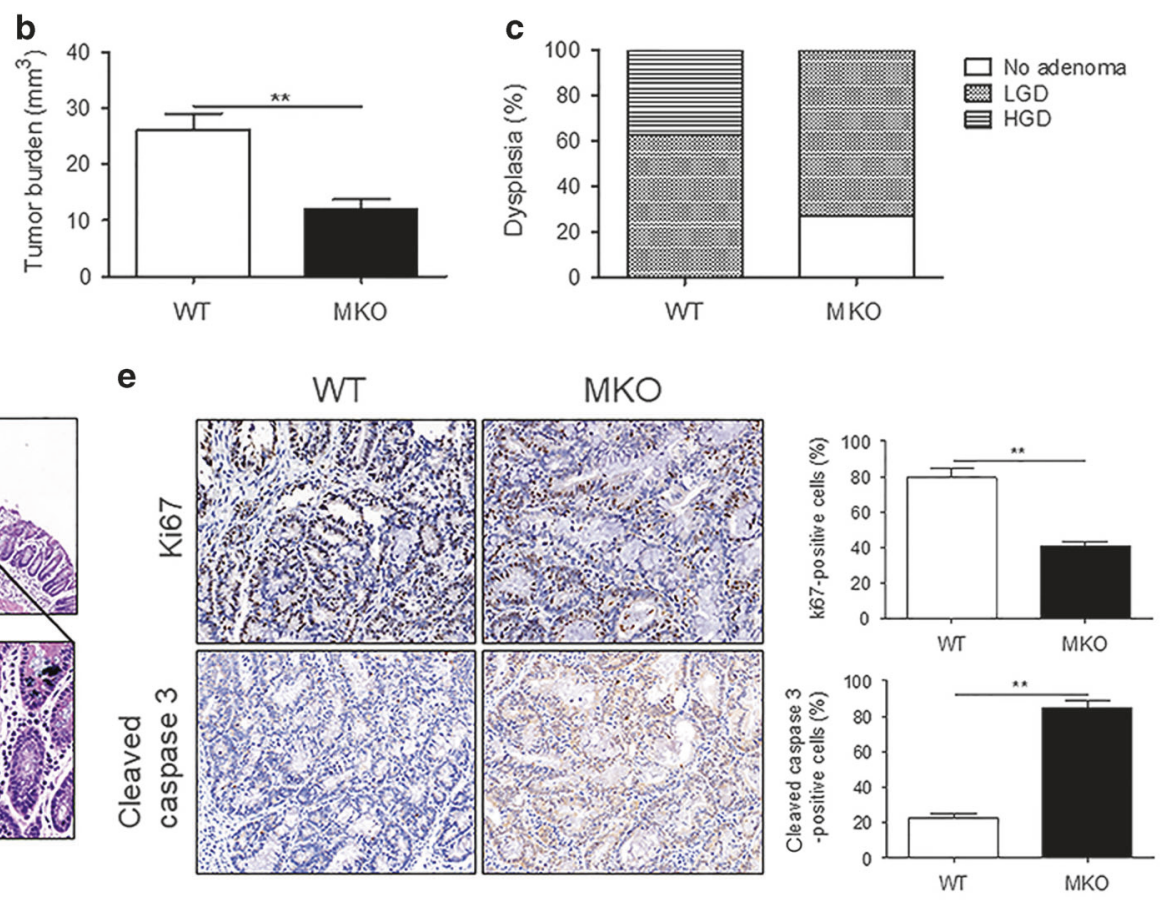

Fig. 2 Myeloid Eftud2 deficiency suppresses inflammatory colon tumorigenesis. a, b On day 100 after CAC induction, tumor number in colon and rectum of MKO and WT mice was counted (a). Tumor burden was determined by calculating the volume of tumor mass (b). Eight pairs of mice were examined. c, d On day 100 after CAC induction, the percentage of cases with either no adenoma, low-grade dysplasia (LGD), and high-grade dysplasia (HGD) was determined (c). H\&E staining was performed (d). Eight pairs of mice were examined. An example is shown. Magnification: upper panel: $\times 100$; lower panel: $\times 400$. e On day 100 following CAC induction, the colonic tissues of MKO and WT mice were dissected. Proliferation and apoptosis of colonic epithelial cells was detected by Ki67 and cleaved caspase 3 staining, respectively. Five pairs of mice were examined. An example is shown on the left and the summarized results on the right. Magnification: $\times 200$. $f$ On day 100 after CAC induction, the colonic tissues of MKO and WT mice were dissected and CEC were isolated as described in Methods. BCl-XL contents in CEC were determined by Western blotting. Each lane represents one mouse. ${ }^{* *} P<0.001$

Eftud2 loss did not affect its level in the colons (Fig. 4b). Similarly, mRNA and protein levels of VEGF-A were increased significantly in the tumors compared with naïve and nontumor colonic tissues. Eftud2 knockout, however, did not impact its expression in the tumors (Fig. 5a, b). In addition, LPS-activated Eftud2-deficient BMDM expressed comparable levels of VEGF-A versus WT BMDM (Fig. $5 \mathrm{C}$ ). These data suggest that Eftud2 is dispensable for the expression of these factors. To further address this issue, we performed immunostaining for CD31, a marker of vascular endothelial cells. Representative images demonstrated significantly enhanced angiogenesis in the tumors versus nontumors, with no difference between WT and MKO tumors (Fig. 5d). The density of $\mathrm{CD}_{3} 1^{+}$blood vessels per case in AOM/DSS-treated MKO mice was comparable to WT controls (Fig. 5e). Thus, diminished tumorigenesis in absence of Eftud2 may not be due to altered angiogenesis.

Eftud2 is required for TLR4 signaling and NF-KB activation in macrophages during intestinal inflammation

TNF- $\mathrm{a}$ is a major inducer of NF-KB activation, and enhanced NF-KB signaling is the hallmark of chronic intestinal inflammation. ${ }^{29,30}$ In addition, Eftud 2 has been previously shown to act as a modulator of TLR4 signaling. ${ }^{17}$ Based on reduced levels of TNF-a in AOM/ DSS-treated MKO mice as described above, we therefore determined the status of activation of TLR4-NF-KB signaling in the lesions during intestinal inflammation and associated tumorigenesis. The activation of TLR4-NF-KB signaling involves IRAK1 hyperphosphorylation, which triggers signaling cascades including IKBa phosphorylation and NF-KB nuclear translocation. Hence, we analyzed phosphorylation of IRAK1, IKKa/ $\beta$, and IKBa in colonic tissues derived from WT and MKO mice on day 35 and 100 post A/D. As shown in Fig. 6a, diminished phosphorylation of IRAK1, IKKa/ $\beta$, and IKBa was observed in MKO compared with WT colon. NF-KB activation was thereby dampened, by detecting the phosphorylation of NF-KB p65 subunit (Fig. 6a). These findings suggest that the alterations of macrophage activation in Eftud2knockout mice may be due to impaired TLR4-NF-KB signaling. To further address this issue, we detected the activation of TLR4-NF$K B$ signaling in LPS-stimulated BMDM in vitro. As shown in Fig. $6 \mathrm{~b}$, the phosphorylation of $1 \mathrm{KKa} / \beta$, IKBa, and NF-KB p65 was apparently decreased in Eftud2-KO BMDM compared with WT controls. In addition, the activation of MAPK ERK1/2 was also inhibited in Eftud2-deficient macrophages (Fig. 6b). Notably, a dramatic reduction in IRAK1 phosphorylation in Eftud2-KO macrophages was readily seen (Fig. 6b), indicating that Eftud2 may primarily regulate AS of the molecules upstream of IRAK1 and thereby enhance the response of macrophages to TLR4 ligation.

Modulation of innate immune responses by Eftud2 involves AS of TLR4/MD-2/MyD88

TLR4, MD-2, and MyD88 are the key molecules upstream of IRAK1 to trigger hyperphosphorylation of IRAK1 upon LPS challenge. 
a

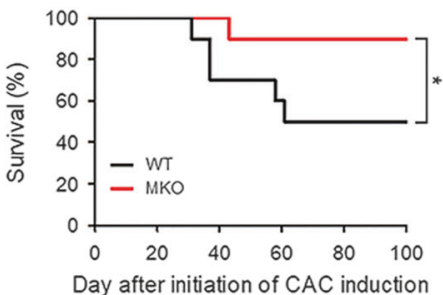

b

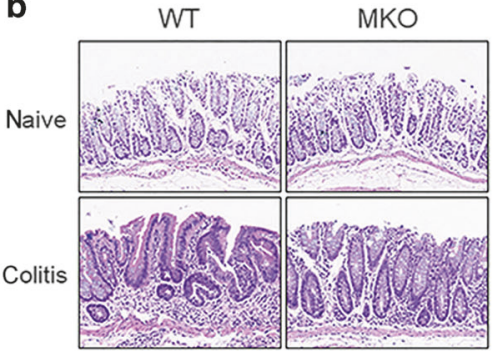

C

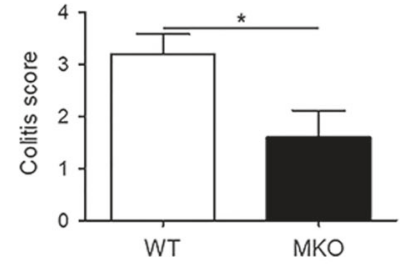

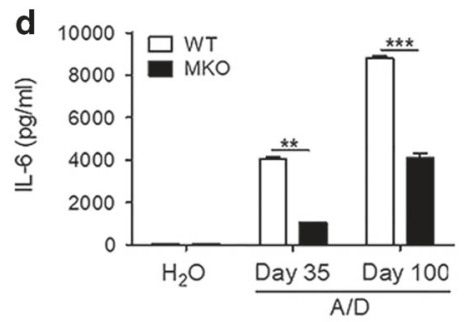

e

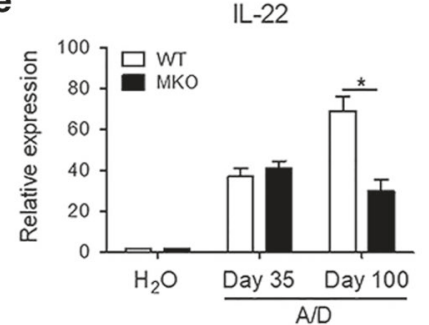

Arg-1

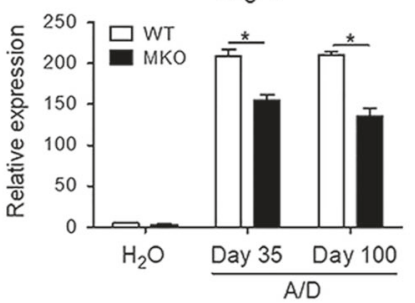

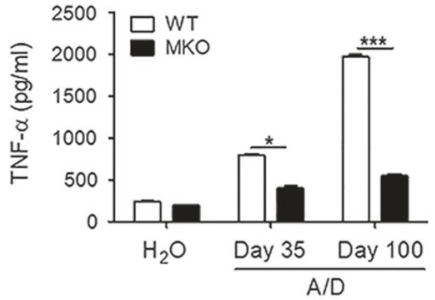

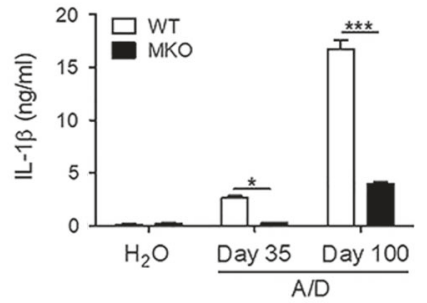

IL-23

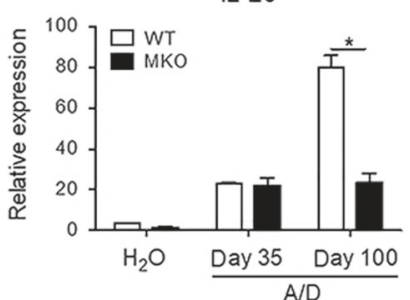

iNOS
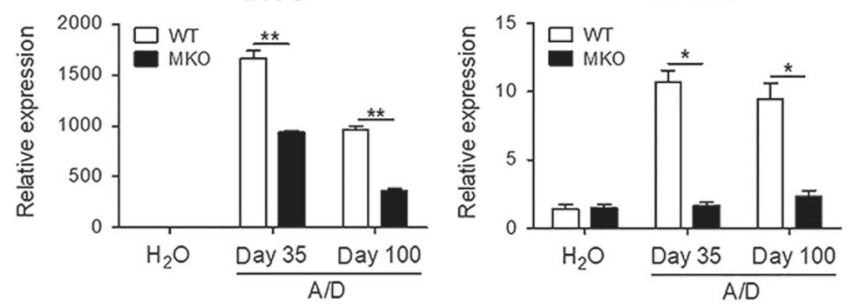

f

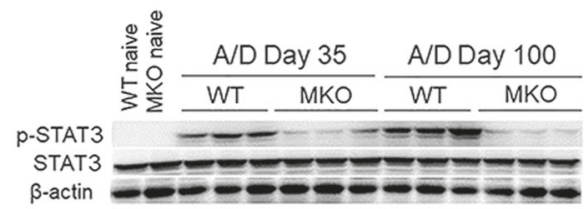

9

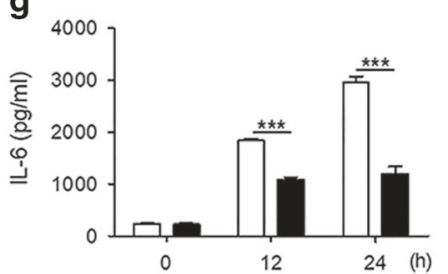

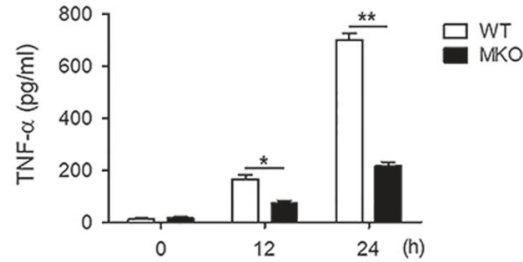

Fig. 3 Loss of Eftud2 in myeloid cells attenuates chronic intestinal inflammation. a CAC models were established in MKO and WT littermates (the number is 20, respectively). Survival rate was addressed by Kaplan-Meier survival analysis. b, c On day 35 after CAC induction, the colons of MKO and WT mice were dissected. H\&E staining was performed. Magnification: $\times 200$ (b). The extent of colitis was scored (c). Five pairs of mice were examined. An example is shown. d On day 35 and 100 following CAC induction, the colons of MKO and WT mice were dissected and cultured for $24 \mathrm{~h}$. The supernatants were collected and cytokine levels indicated were determined by ELISA. Each group consists of $6-8$ mice. e RNA from the colons of MKO and WT mice was extracted and cytokine as well as mediator levels indicated were examined by quantitative RT-PCR. Each group consists of 6-8 mice. f On day 35 and 100 after CAC induction, the colonic tissues of MKO and WT mice were dissected and CEC were isolated as described in Methods. Phosphorylated STAT3 expression in CEC was detected by Western blotting. Each lane represents one mouse. $\mathrm{g}$ BMDM were isolated from MKO and WT mice and cultured as described in Methods. Cells were then stimulated with LPS $(100 \mathrm{ng} / \mathrm{ml})$ for 12 and $24 \mathrm{~h}$, respectively. IL- 6 and TNF- $\alpha$ production were determined by ELISA. The data are pooled from three independent experiments. ${ }^{*} P<0.05$; ${ }^{* *} P<0.01 ;{ }^{* *} P<0.001$

Interestingly, the AS of these molecule mRNAs as a way of regulation of innate immune responses has been recently reported. ${ }^{31-33}$ We thus speculate that the altered splicing of these upstream molecules in Eftud2-deficient macrophages may affect the activation of TLR4 signaling as well as the release of proinflammatory factors. To this end, we detected the expression of long (L) and short (S) forms of MyD88 and MD-2 as well as canonical and soluble isoforms of TLR4 mRNAs in macrophages. LPS challenge enhanced the expression of $L$ and $S$ forms of MyD88 mRNAs and proteins (Figs. 7a and S8A). The levels of MyD88 ${ }_{L}$ in 

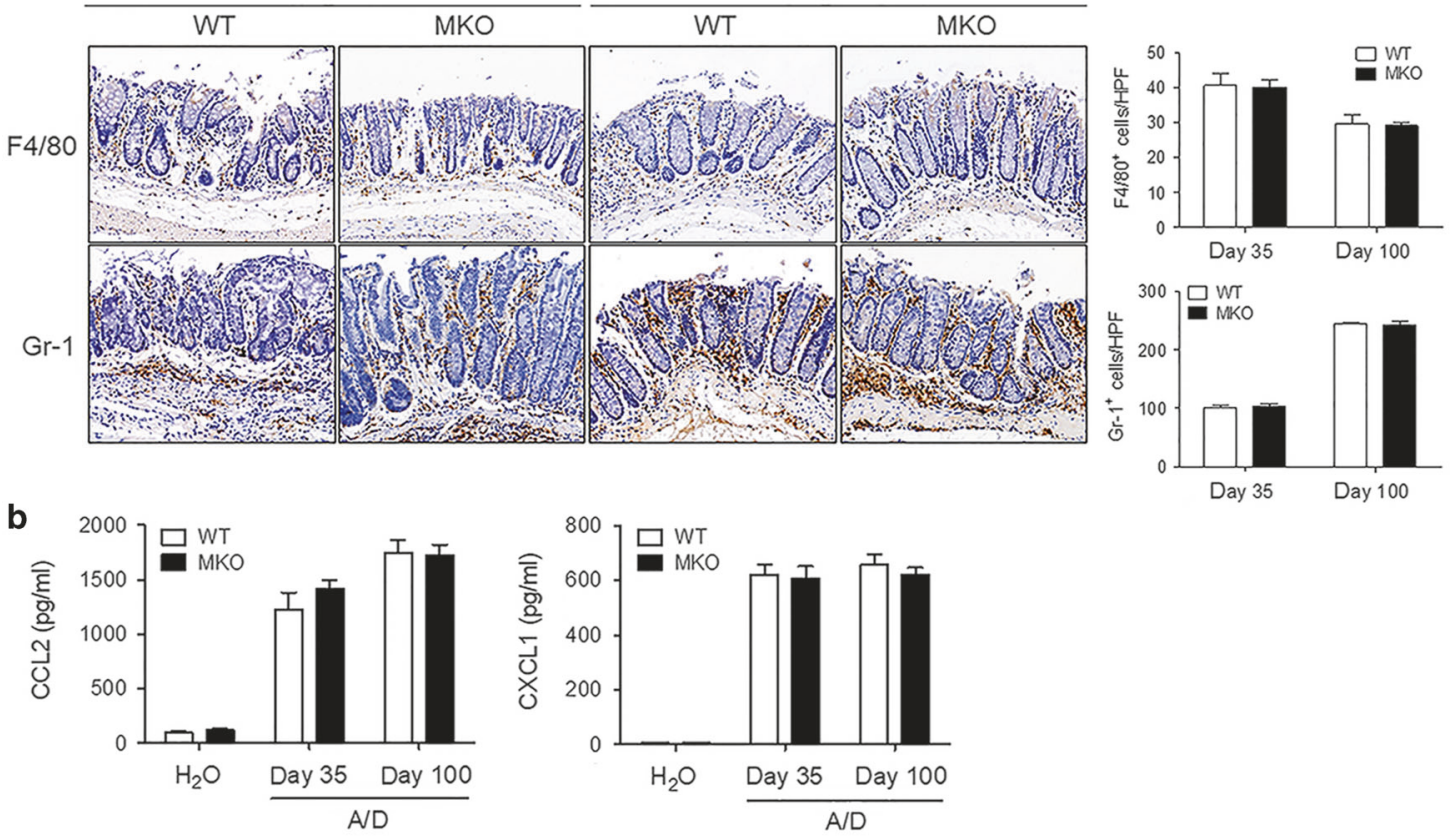

C
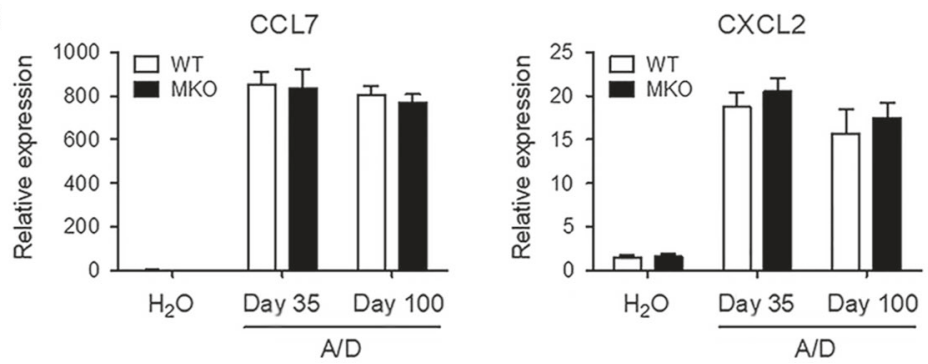

Fig. 4 Myeloid Eftud2 knockout has no impact on macrophage and neutrophil chemotaxis in CAC lesions. a On day 35 and 100 after CAC induction, the colons of MKO and WT mice were dissected and Macrophage $\left(\mathrm{F} 4 / 80^{+}\right)$and neutrophil $\left(\mathrm{Gr}-1^{+}\right)$infiltrates in the colons were detected by immunohistochemistry. Six pairs of mice were analyzed. Left: typical results; Magnification: $\times 200$. Right: summarized results; HPF, high-power field. b, c On day 35 and 100 after CAC induction, the colons of MKO and WT mice were dissected and the levels of chemokines indicated in the colons were determined by ELISA (b) and quantitative RT-PCR (c). The data were pooled from two independent experiments. Each group consists of 6-8 mice

Eftud2-KO macrophages, however, were significantly lower than that in WT controls (Figs. 7a and S8A). In a sharp contrast, MyD88, expression was dramatically increased in Eftud2-deficient macrophages (Figs. 7a and S8A). Importantly, these changes were also observed in the tumors of AOM/DSS-treated MKO mice versus WT littermates (Fig. 7b). Intriguingly, similar to MyD88, the altered splicing of TLR4 and MD-2 was found in Eftud2-KO macrophages, as well as in the colon tumors (Fig. S8A-E). These data suggest that altered splicing of mRNAs of these molecules may in whole or in part explain the weakened response to LPS when Eftud2 is deleted. To further address this, we used specific siRNA to knockdown the expression of spliced isoforms of MyD88/TLR4/ MD-2 in combination or alone in Eftud2-KO macrophages, which did not affect the expression of canonical isoforms of these molecules (Fig. 7c). The treatment of cells with this mixture of MyD88s/sTLR4/MD-2B-specific siRNA in Eftud2-KO macrophages rendered a remarkable increase in LPS-induced IL-6, TNF- $a$, and IL$1 \beta$ expression (Fig. 7c). This effect was due to partial restoration of TLR4-NF-KB signaling activation in Eftud2-KO macrophages (Fig. 7d). To further directly address the effect of Eftud2mediated splicing on NF-KB activation, a luciferase assay for NF$\kappa B$ reporter in MyD88s/sTLR4/MD-2B-silenced MKO BMDM was undertaken. The treatment of Eftud2-KO macrophages with
MyD88s/sTLR4/MD-2B-specific siRNA alone or in combination led to a large increase in reporter activity upon LPS challenge, which was most pronounced in siRNA mixture-treated BMDM (Fig. S9). These data clearly suggest that the NF-KB pathway is regulated by Eftud2-mediated splicing of molecules involving TLR4 signaling.

\section{DISCUSSION}

Eftud2 is a component of the U5 snRNP in the spliceosome that mediates pre-mRNA splicing, whose mutations in human cause craniofacial conditions including mandibulofacial dysostosis and oesophageal atresia. ${ }^{16,34}$ Recently, Alper and colleagues have taken a comparative genomics RNAi screening in the nematode $C$. elegans and murine macrophages and identified Eftud 2 as a novel regulator of innate immunity. ${ }^{17}$ Furthermore, Eftud 2 represents a key factor to modulate innate immune response to virus infection by regulating AS of RIG-I, which underlines the important role of Eftud2 in innate immunity. ${ }^{35}$ However, its role in chronic inflammation and the associated tumorigenesis remains elusive. This report defined for the first time the tumor-promoting function of Eftud2 on colitis-associated tumorigenesis by modulating inflammatory phenotypes of macrophages. 


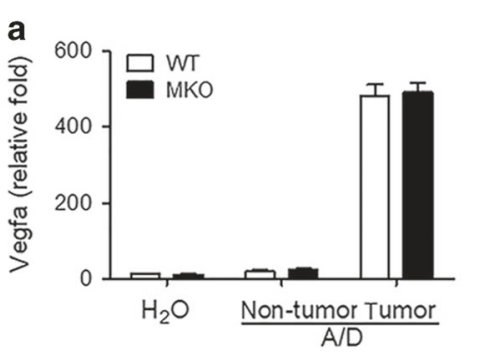

d

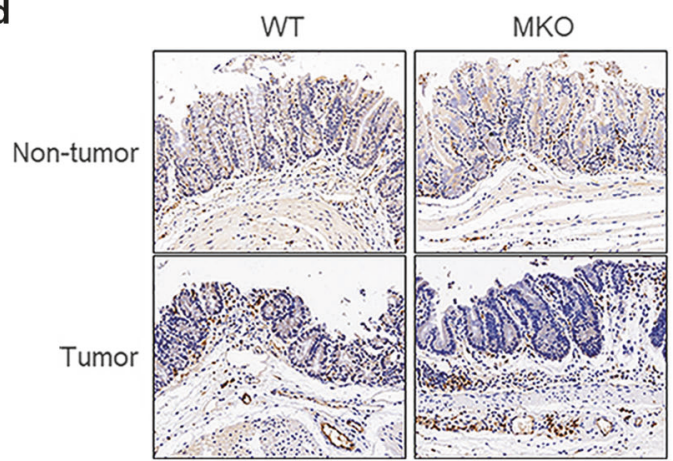

b

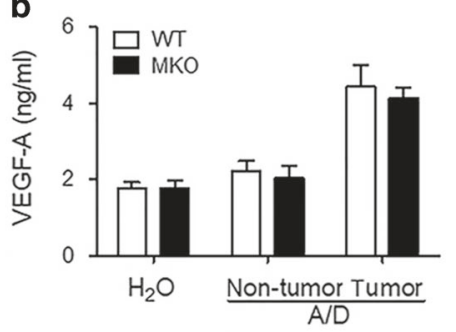

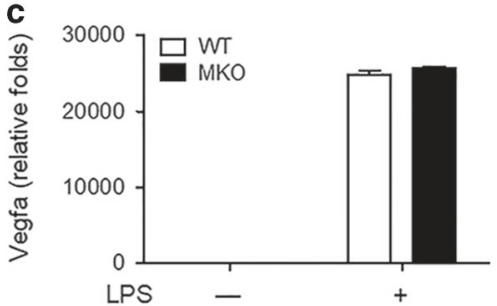

e

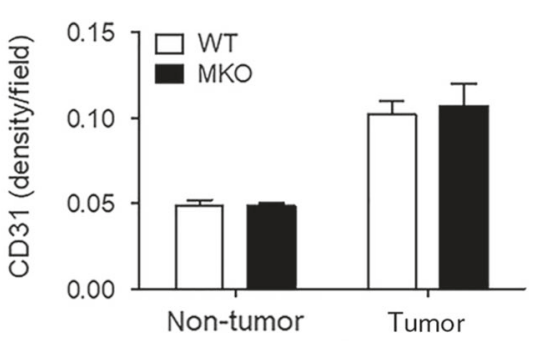

Fig. 5 Myeloid Eftud2 deletion does not affect angiogenesis during colon tumorigenesis. a, b On day 100 after CAC induction, the levels of VEGF-A in the colons of MKO and WT mice were determined by quantitative RT-PCR (a) and ELISA (b). Each group consists of 5-6 mice. c BMDM were isolated from MKO and WT mice and stimulated with LPS $(100 \mathrm{ng} / \mathrm{ml})$ for $6 \mathrm{~h}$. VEGF-A expression was detected by quantitative RT-PCR. The data are pooled from two independent experiments. d, e On day 100 following CAC induction, the colons of MKO and WT mice were dissected and blood vessels $\left(\mathrm{CD} 1^{+}\right)$were detected by immunohistochemistry. Five pairs of mice were analyzed. Typical results were shown (d). Magnification: $\times 200$. Staining density was quantified as described in supplementary methods (e)

The increase in mucosal macrophage population is well documented in UC patients. ${ }^{36}$ Recruitment and activation of macrophages within the intestinal mucosa play a key role in the pathogenesis of both human UC $\mathrm{C}^{36,37}$ and murine DSS-induced colitis. ${ }^{38,39}$ A large number of studies suggest that activated macrophages are candidate linking cells between inflammation and cancer. ${ }^{40,41}$ For instance, the tumor-promoting cytokines IL-1, IL-6, and TNF-a are produced mainly by activated macrophages and, along with macrophage-derived growth factor and reactive oxygen species, foster colitis-associated tumorigenesis. ${ }^{37,40}$ These factors have potentials to enhance the proliferation of intestinal epithelial cells by activating STAT3 and its downstream target genes and/or upregulating COX-2 expression. ${ }^{5,6,18}$ Interestingly, $\mathrm{Bcl}-\mathrm{XL}$ has been reported previously to be a target gene of STAT3 in human and murine cancers, ${ }^{42}$ suggesting that one of the mechanisms underlying IL-6/STAT3-mediated tumor growth is to augment $\mathrm{BCl}-\mathrm{XL}$ expression in the colonic epithelium. Therefore, a drastic reduction in production of these cytokines and other growth factors in activated macrophages as well as decreased STAT3 activation and BCl-XL contents in epithelium strongly support our phenotypic observation and explain the mechanism of reduced tumor incidence in Eftud2-deficient mice.

One of the unique aspects of colorectal cancer development is the involvement of luminal flora and TLR signaling, among which the ligand for TLR4 (i.e., LPS) is abundant in the colonic lumen and colonic bacteria and induce growth of colonic tumors through activation of TLR4. ${ }^{8}$ TLR4 expression was found in both epithelial cells as well as lamina propria macrophages in neoplastic areas. NF-kB, which is well-known to be critical for both mouse model and human colorectal tumorigenesis, can be activated by many upstream stimuli including TLRs. In the setting of intestinal inflammation, TLR4 may be a principal receptor, upstream of $\mathrm{NF}-\mathrm{KB}$, promoting the development of colonic tumors. This concept coincides with our observations of the impaired activation of TLR4-NF-KB signaling pathway in Eftud2-KO macrophages and the colonic tissues of Eftud2-deficient mice. Notably, the activation of MAPK/ERK was also abolished in the colons of AOM/DSS-treated MKO mice as well as LPS-challenged Eftud2-KO macrophages, which is in line with recent studies that ERK activation is an integral part of tumor development in a mouse model of colorectal tumorigenesis. ${ }^{43}$ Given the importance of NF-KB and ERK signaling pathway in initiation and maintenance of chronic intestinal inflammation and malignant transformation, it is thus reasonable that myeloid loss of Eftud2 results in the impairment of NF-KB and ERK activation in macrophages and thereby diminish the release of proinflammatory cytokines and growth factors, and ultimately suppresses tumor development and progression.

It is known that TLR4 signaling is controlled by hosts at multiple layers to maintain homeostatic states, among which AS is an important approach by regulating the splicing of adaptors and kinases, including TLR4, MD-2 and MyD88, although the mechanisms by which the spliced isoforms of TLR4/MD-2/MyD88 regulate the downstream TLR4 signaling remain incompletely known. LPS stimulation increases the mRNA of soluble form of TLR4 in murine macrophages that inhibits TNF-a production and NF-KB activation, likely by disrupting TLR4-MD-2 interaction or interacting with the newly synthesized TLR4 or CD14 in the membrane. ${ }^{31}$ An alternatively spliced form of MD-2, which lacks the first 54 bases of exon 3, competes with full-length MD-2 for binding to TLR4 and decreases the number of TLR4/MD-2 complexes on the cell surface, resulting in the inhibition of LPS signaling. ${ }^{32} \mathrm{MyD}_{\mathrm{s}}$, an alternatively spliced form of MyD88 ${ }_{L}$, lacks only the short intermediate domain separating the $\mathrm{N}$-terminal death domain and $\mathrm{COOH}$-terminal Toll/IL-1 receptor (TIR) domains. Mechanistically, MyD88 s has potentials to bind to the TLR signaling complex and can still interacts with IRAK1. However, MyD88 s cannot interact with IRAK4, leading to loss of phosphorylation and activation of IRAK1 and consequently a shutdown of IL-1/LPSinduced NF-KB activation. ${ }^{33,44,45}$ A previous study has shown that MyD88 splicing was involved in the regulation of TLR4 signaling by Eftud2. ${ }^{17}$ In this study, we further demonstrated that Eftud2 affected the splicing of TLR4/MyD88/MD-2 and that combined 
a

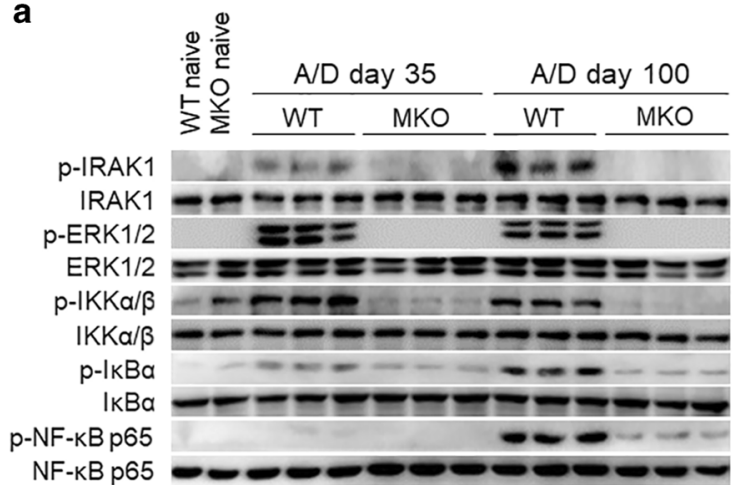

b

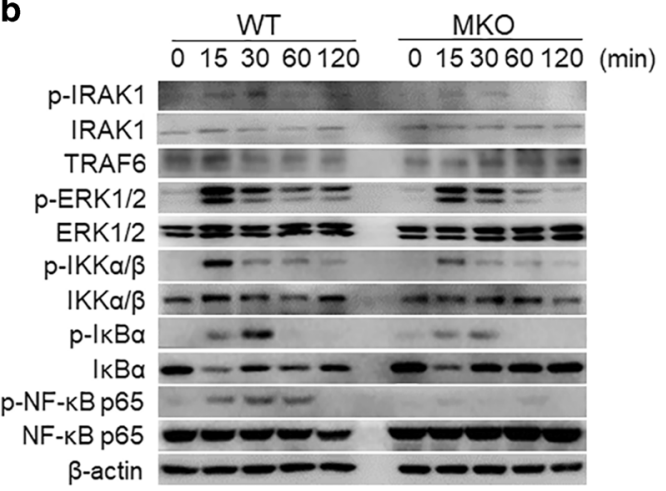

Fig. 6 Decreased LPS/TLR4 signaling pathway in MKO mice subjected to AOM/DSS. a On day 35 and 100 after CAC induction, the colons of MKO and WT mice were dissected and the expression of the kinases and proteins indicated involving TLR4 signaling in the colons was detected by Western blotting. Each lane represents one mouse. b BMDM were isolated from MKO and WT mice and treated with LPS (100 ng/ml) for 0-2 $h$ and the activation of TLR4-NF-KB signaling was detected by Western blotting. An example of four independent experiments is shown

knockdown of spliced isoforms of TLR4/MyD88/MD-2 had most profound effects on unleashing the suppression of innate responses in Eftud2-deficient macrophages, indicating that deregulated splicing of MyD88/TLR4/MD-2 mRNAs contributes to the inhibition of TLR4 signaling and NF-KB activation. Whether there are altered splicing of mRNA of one or multiple molecules (except those discussed above) involving TLR4 signaling and NF$\mathrm{KB}$ activation in absence of Eftud2 is not addressed in this study, which need further investigations by RNA sequencing.

It is worthy to note that, although proinflammatory cytokine release was diminished, loss of Eftud 2 in macrophages did not affect overall cell viability, chemotaxis, and phagocytic functions as well as angiogenesis. This suggests that at the level of gene inhibition, the innate immune response is far more sensitive to Eftud2 levels than other general cell functions. Future investigations are warranted to determine Eftud 2 expression in samples of human colorectal cancer and define the clinical correlations of Eftud2 with immune cell infiltration, proinflammatory/pro-angiogenic factor expression as well as patient's outcome. The deeply understanding of actions of Eftud2 on colorectal cancer development might provide new insights into the underlying mechanisms of AS to shape tumor immune microenvironment and establish the crosstalk between cancer and host immunity.

\section{METHODS}

Mice

Eftud2-floxed mice on C57/BL6 background were developed by Cyagen Biosciences (Guangzhou, China). The targeted strategy is illustrated in Fig. S2A. The neo ${ }^{R}$ cassette was removed by crossing Eftud2-floxed founders with FLP delete strain. Cre-mediated recombination resulted in the deletion of exon 5-7 and subsequent frameshift of the Eftud2 gene. Myeloid lineagespecific Eftud2 knockout mice were generated by crossing the Eftud2-floxed mice with LysM-Cre recombinase transgenic mice (Model Animal Research Center of Nanjing University, China), the wild-type (WT) littermates (LysM-Cre negative, Eftud2 ${ }^{\mathrm{f} / \mathrm{fl}}$ ) were used as controls. Male C57BL/6 mice at 6-8 weeks of age were purchased from Beijing Vital River Laboratory Animal Technology Co. Ltd., China. Animals were housed in specific pathogen-free conditions with an alternating light/dark cycle. Care, use, and treatment in this study were in strict agreement with international guidelines for the care and use of laboratory animals and approved by Animal Ethics Committee of State Key Laboratory of Toxicology and Medical Countermeasures.
Acute colitis and CAC induction

Acute colitis model was established by drinking 3\% dextran sulfate sodium (MW 36,000-50,000; MP Biochemicals) for 7 days. CAC was induced according to the protocols as described in our previous study ${ }^{46}$ (Fig. 1a). The details are described in Supplementary methods.

\section{Histolopathology}

Formalin-preserved colon sections were processed and embedded in paraffin by standard techniques. Longitudinal sections of $5-\mu \mathrm{m}$ thick were stained with hematoxylin and eosin (H\&E) and examined by a pathologist blinded to the experimental groups. Colitis was scored according to the criteria as previously described. ${ }^{47}$ The protocols on immunohistochemistry are described in Supplementary methods.

Isolation of CEC

CEC were fractionated as described previously ${ }^{48}$ with the purity $>80 \%$, identified by CK-18 staining.

Isolation of lamina propria immune cells

Immune cells in lamina propria of the colon were isolated according to the protocol described by our previous study. ${ }^{49}$

Induction of sterile peritonitis

Mice were injected i.p. with Brewer thioglycollate medium (4\%; Solarbio). Three days later, mice were sacrificed for collection of peritoneal cavity cells and peripheral blood to be used for flow cytometry.

Macrophage phagocytosis examination

Experiments were carried out as described in Supplementary methods.

Immuoblotting

Standard procedures for immunoblotting are described in Supplementary methods.

Whole colon culture

The protocols are described in Supplementary methods.

Cytokine and chemokine analysis

Cytokines and chemokines were examined by ELISA kits obtained from R\&D systems or Biolegend according to the manufacturer's instructions. 
a
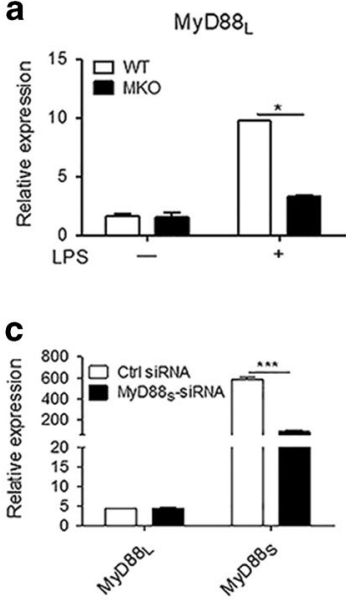

IL-6

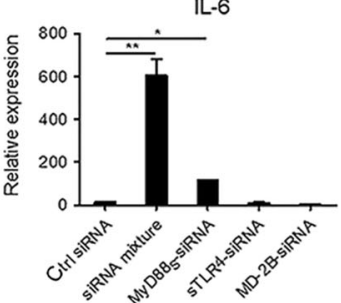

MyD88s
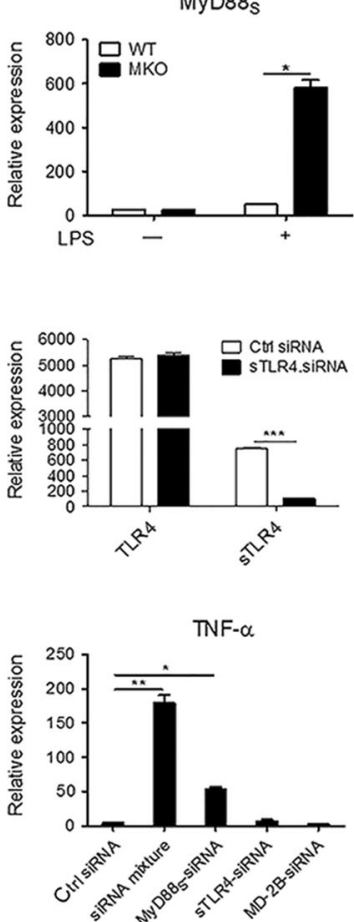

b
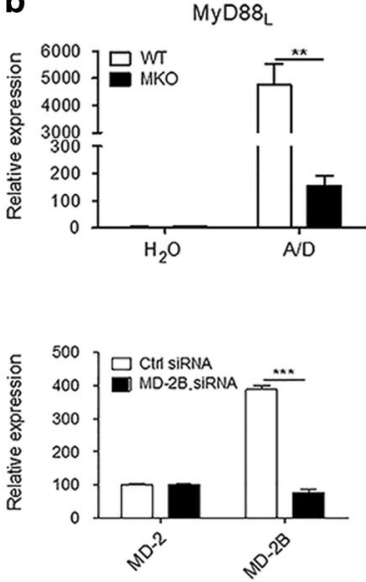

IL-1 $\beta$

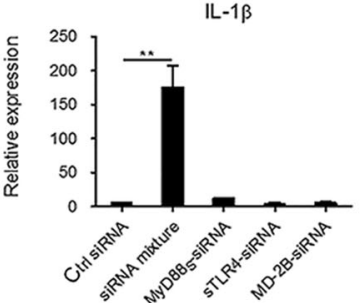

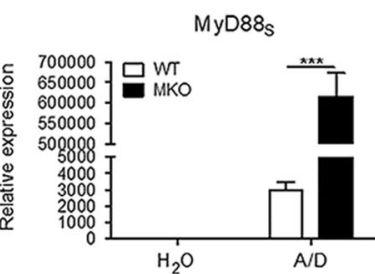

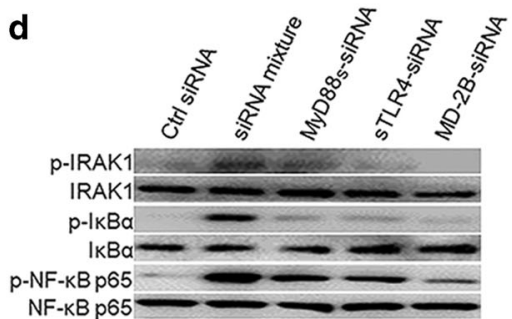

Fig. 7 AS is involved in the control of LPS/TLR4 signals by Eftud2. a BMDM were isolated from MKO and WT mice and treated with LPS $(100 \mathrm{ng} / \mathrm{ml})$ for $2 \mathrm{~h}$. The expression of $\mathrm{L}$ and $\mathrm{S}$ forms of MyD88 was examined by quantitative RT-PCR. An example of five independent experiments is shown. b On day 100 after CAC induction, the levels of $L$ and S forms of MyD88 mRNA in the colons of MKO and WT mice were determined by quantitative RT-PCR. Six pairs of mice were analyzed. c Eftud2-KO BMDM were transfected by MyD88 $/$ sTLR4/MD-2B siRNA alone or in combination and treated with LPS $(100 \mathrm{ng} / \mathrm{ml})$ for $6 \mathrm{~h}$. The levels of canonical and spliced isoforms of MyD88/TLR4/MD-2 as well as Cytokine expression indicated was determined by quantitative RT-PCR. An example of three independent experiments is shown. $\mathbf{d}$ MyD88 $/$ sTLR4/MD-2B-knockdown Eftud2-deficient BMDM were treated with LPS (100 ng/ml) for $30 \mathrm{~min}$. The activation of TLR4 signaling was detected by Western blotting. An example of three independent experiments is shown. ${ }^{* *} P<0.01$

Cell line, macrophage preparation, and treatment

Murine macrophage cell line RAW264.7 was purchased from ATCC and maintained in RPMI-1640 medium supplemented with $10 \% \mathrm{FBS}, 100 \mathrm{U} / \mathrm{ml}$ penicillin, and $100 \mathrm{mg} / \mathrm{ml}$ streptomycin. For primary macrophage preparation, bone marrow cells were obtained from the femurs of mice. Single-cell suspensions were prepared following res cell lysis in RPMI-1640 medium. Bone marrow cells were cultured in complete RPMI-1640 medium for $6 \mathrm{~h}$, and floating cells were removed to enrich monocytes. BMDM were generated by treating monocytes with recombinant murine M-CSF ( $25 \mathrm{ng} / \mathrm{ml}$; Peprotech) for 7 days. For obtaining peritoneal macrophages, peritoneal exudate cells were cultured in RPMI-1640 medium for $4 \mathrm{~h}$, and the attached cells were collected. Macrophage purity (>90\%) was validated by flow cytometry. Macrophages were seeded in six-well cultured plates $\left(1 \times 10^{6} / \mathrm{ml} /\right.$ well) and stimulated with LPS (Sigma-Aldrich) at indicated concentrations for $2-24 \mathrm{~h}$. The supernatants or cells were collected for use.

Cell viability assay

Experiments were carried out as described in Supplementary methods.

\section{Flow cytometry}

The protocols are described in Supplementary methods.

RNA silencing and reporter gene assay

Experiments were carried out as described in Supplementary methods.
Quantitative RT-PCR

The protocols are described in Supplementary methods. The primers for realtime were listed in Supplemental Table.

Statistical analysis

Data are presented as the mean \pm SD. Student's $t$ test (paired or unpaired) and one-way analysis of variance (ANOVA) were used to determine significance, with a $P<0.05$ being considered significant. Statistical tests were performed using SPSS 10.0 for Macintosh, and graphs were prepared using Deltagraph (SPSS, Chicago, 111.).

\section{ACKNOWLEDGEMENTS}

This work is supported by grants from the National Key Basic Research Program of China (2015CB553704), the National Natural Science Foundation of China (81672803, $81472647,81272320,81773755,31500732)$, and the Zhejiang Technology Program of Laboratory Animals (2018C37128).

\section{AUTHOR CONTRIBUTIONS}

Study concept and design: Y.C. and G.C. Acquisition of data: Z.L., Z.W., L.L., Y.C., R.W., H.X., Y.W. and G.C. Analysis and interpretation of data: Z.L., Z.W., G.H., J.F., B.S., H.P., Y.L. and G.C. Drafting of the manuscript: Z.L. and G.C. Critical revision of the manuscript for important intellectual content: R.G., B.S., and Y.L. Statistical analysis: Z.L. and Z.W. Obtained funding: Y.C., Y.W., H.P. and G.C. Administrative, technical, or material support: X.L. and C.H. 


\section{ADDITIONAL INFORMATION}

The online version of this article (https://doi.org/10.1038/s41385-019-0184-y) contains supplementary material, which is available to authorized users.

Competing interests: The authors declare no competing interests.

Publisher's note: Springer Nature remains neutral with regard to jurisdictional claims in published maps and institutional affiliations.

\section{REFERENCES}

1. Eaden, J. A., Abrams, K. R. \& Mayberry, J. F. The risk of colorectal cancer in ulcerative colitis: a meta-analysis. Gut 48, 526-535 (2001).

2. Fiocchi, C. Inflammatory bowel disease: etiology and pathogenesis. Gastroenterology 115, 182-205 (1998).

3. Fantini, M. C. \& Pallone, F. Cytokines: from gut inflammation to colorectal cancer. Curr. Drug Targets 9, 375-380 (2008).

4. Ostuni, R., Kratochvill, F., Murray, P. J. \& Natoli, G. Macrophages and cancer: from mechanisms to therapeutic implications. Trends Immunol. 36, 229-239 (2015).

5. Grivennikov, S. et al. IL-6 and Stat3 are required for survival of intestinal epithelial cells and development of colitis-associated cancer. Cancer Cell 15, 103-113 (2009).

6. Popivanova, B. K. et al. Blocking TNF-alpha in mice reduces colorectal carcinogenesis associated with chronic colitis. J. Clin. Invest 118, 560-570 (2008).

7. Kaler, P., Augenlicht, L. \& Klampfer, L. Macrophage-derived IL-1beta stimulates Wnt signaling and growth of colon cancer cells: a crosstalk interrupted by vitamin D3. Oncogene 28, 3892-3902 (2009).

8. Fukata, M. et al. Toll-like receptor-4 promotes the development of colitisassociated colorectal tumors. Gastroenterology 133, 1869-1881 (2007).

9. Leu, C. M., Wong, F. H., Chang, C., Huang, S. F. \& Hu, C. P. Interleukin-6 acts as an antiapoptotic factor in human esophageal carcinoma cells through the activation of both STAT3 and mitogen-activated protein kinase pathways. Oncogene 22, 7809-7818 (2003).

10. Stumpo, D. J., Lai, W. S. \& Blackshear, P. J. Inflammation: cytokines and RNA-based regulation. Wiley Interdiscip. Rev. RNA 1, 60-80 (2010).

11. O'Connell, R. M., Rao, D. S. \& Baltimore, D. MicroRNA regulation of inflammatory responses. Annu. Rev. Immunol. 30, 295-312 (2012).

12. Pandya-Jones, A. et al. Splicing kinetics and transcript release from the chromatin compartment limit the rate of Lipid A-induced gene expression. RNA 19, 811-827 (2013).

13. O'Connor, B. P. et al. Regulation of toll-like receptor signaling by the SF3a mRNA splicing complex. PLoS Genet. 11, e1004932 (2015)

14. de Bruin, R. G. et al. Quaking promotes monocyte differentiation into proatherogenic macrophages by controlling pre-mRNA splicing and gene expression. Nat. Commun. 7, 10846 (2016)

15. Lin, J. et al. Transcriptome-wide analysis reveals modulation of human macrophage inflammatory phenotype through alternative splicing. Arterioscler. Thromb. Vasc. Biol. 36, 1434-1447 (2016).

16. Gordon, C. T. et al. EFTUD2 haploinsufficiency leads to syndromic oesophageal atresia. J. Med. Genet. 49, 737-746 (2012).

17. De Arras, L. et al. Comparative genomics RNAi screen identifies Eftud2 as a novel regulator of innate immunity. Genetics 197, 485-496 (2014).

18. Matsumoto, S. et al. Essential roles of IL- 6 trans-signaling in colonic epithelia cells, induced by the IL-6/soluble-IL-6 receptor derived from lamina propria macrophages, on the development of colitis-associated premalignant cancer in a murine model. J. Immunol. 184, 1543-1551 (2010).

19. Rakoff-Nahoum, S., Paglino, J., Eslami-Varzaneh, F., Edberg, S. \& Medzhitov, R. Recognition of commensal microflora by toll-like receptors is required for intestinal homeostasis. Cell 118, 229-241 (2004)

20. Francescone, R., Hou, V., \& Grivennikov, S. I. Cytokines, IBD, and colitis-associated cancer. Inflamm. Bowel Dis. 21, 409-418 (2015).

21. Yu, H., Pardoll, D. \& Jove, R. STATs in cancer inflammation and immunity: a leading role for STAT3. Nat. Rev. Cancer 9, 798-809 (2009).

22. Rmali, K. A., Puntis, M. C. \& Jiang, W. G. Tumour-associated angiogenesis in human colorectal cancer. Colorectal Dis. 9, 3-14 (2007).

23. Mousa, L., Salem, M. E. \& Mikhail, S. Biomarkers of angiogenesis in colorectal cancer. Biomark. Cancer 7, 13-19 (2015).

24. Li, A., Dubey, S., Varney, M. L., Dave, B. J. \& Singh, R. K. IL-8 directly enhanced endothelial cell survival, proliferation, and matrix metalloproteinases production and regulated angiogenesis. J. Immunol. 170, 3369-3376 (2003).

25. Heidemann, J. et al. Angiogenic effects of interleukin 8 (CXCL8) in human intestinal microvascular endothelial cells are mediated by CXCR2. J. Biol. Chem. 278, 8508-8515 (2003)
26. Angelo, L. S. \& Kurzrock, R. Vascular endothelial growth factor and its relationship to inflammatory mediators. Clin. Cancer Res. 13, 2825-2830 (2007).

27. Wu, W. K., Llewellyn, O. P., Bates, D. O., Nicholson, L. B. \& Dick, A. D. IL-10 regulation of macrophage VEGF production is dependent on macrophage polarisation and hypoxia. Immunobiology 215, 796-803 (2010).

28. Engstrom, A., Erlandsson, A., Delbro, D. \& Wijkander, J. Conditioned media from macrophages of $M 1$, but not $M 2$ phenotype, inhibit the proliferation of the colon cancer cell lines HT-29 and CACO-2. Int. J. Oncol. 44, 385-392 (2014).

29. Greten, F. R. et al. IKKbeta links inflammation and tumorigenesis in a mouse model of colitis-associated cancer. Cell 118, 285-296 (2004).

30. Karin, M. \& Greten, F. R. NF-kappaB: linking inflammation and immunity to cancer development and progression. Nat. Rev. Immunol. 5, 749-759 (2005).

31. Iwami, K. I. et al. Cutting edge: naturally occurring soluble form of mouse toll-like receptor 4 inhibits lipopolysaccharide signaling. J. Immunol. 165, 6682-6686 (2000).

32. Ohta, S., Bahrun, U., Tanaka, M. \& Kimoto, M. Identification of a novel isoform of MD-2 that downregulates lipopolysaccharide signaling. Biochem. Biophys. Res. Commun. 323, 1103-1108 (2004).

33. Burns, K. et al. Inhibition of interleukin 1 receptor/Toll-like receptor signaling through the alternatively spliced, short form of MyD88 is due to its failure to recruit IRAK-4. J. Exp. Med. 197, 263-268 (2003).

34. Sarkar, A. et al. Novel de novo mutations in EFTUD2 detected by exome sequencing in mandibulofacial dysostosis with Microcephaly syndrome. Am. J. Med. Genet. Part A 167A, 914-918 (2015).

35. Zhu, C. et al. EFTUD2 is a novel innate immune regulator restricting hepatitis $C$ virus infection through the RIG-I/MDA5 Pathway. J. Virol. 89, 6608-6618 (2015).

36. Mahida, Y. R. The key role of macrophages in the immunopathogenesis of inflammatory bowel disease. Inflamm. Bowel Dis. 6, 21-33 (2000).

37. Sanchez-Munoz, F., Dominguez-Lopez, A. \& Yamamoto-Furusho, J. K. Role of cytokines in inflammatory bowel disease. World J. Gastroenterol. 14, 4280-4288 (2008).

38. Elson, C. O., Sartor, R. B., Tennyson, G. S. \& Riddell, R. H. Experimental models of inflammatory bowel disease. Gastroenterology 109, 1344-1367 (1995).

39. Krieglstein, C. F. et al. Collagen-binding integrin alpha1beta1 regulates intestinal inflammation in experimental colitis. J. Clin. Investig. 110, 1773-1782 (2002).

40. Coussens, L. M. \& Werb, Z. Inflammation and cancer. Nature 420, 860-867 (2002).

41. Pollard, J. W. Tumour-educated macrophages promote tumour progression and metastasis. Nat. Rev. Cancer 4, 71-78 (2004).

42. Catlett-Falcone, R. et al. Constitutive activation of Stat3 signaling confers resistance to apoptosis in human U266 myeloma cells. Immunity 10, 105-115 (1999).

43. Lee, S. H. et al. ERK activation drives intestinal tumorigenesis in $\mathrm{Apc}(\mathrm{min} /+)$ mice. Nat. Med. 16, 665-670 (2010).

44. Burns, K. et al. MyD88, an adapter protein involved in interleukin-1 signaling. J. Biol. Chem. 273, 12203-12209 (1998).

45. Janssens, S., Burns, K., Tschopp, J. \& Beyaert, R. Regulation of interleukin-1- and lipopolysaccharide-induced NF-kappaB activation by alternative splicing of MyD88. Curr. Biol. 12, 467-471 (2002).

46. Wang, Y. et al. Tumor-derived GM-CSF promotes inflammatory colon carcinogenesis via stimulating epithelial release of VEGF. Cancer Res. 74, 716-726 (2014).

47. Wang, K. et al. Opposite role of tumor necrosis factor receptors in dextran sulfate sodium-induced colitis in mice. PloS ONE 7, e52924 (2012).

48. Guo, J., Longshore, S., Nair, R. \& Warner, B. W. Retinoblastoma protein (pRb), but not p107 or p130, is required for maintenance of enterocyte quiescence and differentiation in small intestine. J. Biol. Chem. 284, 134-140 (2009).

49. Wang, Y. et al. Neutrophil infiltration favors colitis-associated tumorigenesis by activating the interleukin-1 (IL-1)/IL-6 axis. Mucosal. Immunol. 7, 1106-1115 (2014)

Open Access This article is licensed under a Creative Commons Attribution 4.0 International License, which permits use, sharing adaptation, distribution and reproduction in any medium or format, as long as you give appropriate credit to the original author(s) and the source, provide a link to the Creative Commons license, and indicate if changes were made. The images or other third party material in this article are included in the article's Creative Commons license, unless indicated otherwise in a credit line to the material. If material is not included in the article's Creative Commons license and your intended use is not permitted by statutory regulation or exceeds the permitted use, you will need to obtain permission directly from the copyright holder. To view a copy of this license, visit http://creativecommons. org/licenses/by/4.0/.

(c) The Author(s) 2019 